\title{
Evolution of Mixed Particles Interacting with Classical Sources
}

\author{
Maxim Dvornikov \\ Department of Physics, P.O. Box 35, FIN-40014, University of Jyväskylä, Finland \\ IZMIRAN, 142190, Troitsk, Moscow region, Russia and \\ Departamento de Física y Centro de Estudios Subatómicos, \\ Universidad Técnica Federico Santa María, Casilla 110-V, Valparaíso, Chile
}

(Dated: October 18, 2018)

\begin{abstract}
We study the systems of scalar and spinor particles with mixing emitted by external classical sources. The particles wave functions exactly accounting for external sources are obtained directly from the Lorentz invariant wave equations in (3+1)-dimensional space-time. Then we discuss sources which are localized in space and emit harmonic radiation. We obtain that the considered scalar and spinor fields can be converted from one type to another due to the presence of the vacuum mixing. This phenomenon is shown to be analogous to neutrino flavor oscillations in vacuum since the calculated transition and survival probabilities coincide with the corresponding expressions for neutrino oscillations. When we discuss the evolution of spinor particles, the situation of general mass matrix, which has both Dirac and Majorana mass terms, is studied. In this case we analyze the appearance of antiparticles in the initial particles beam. The relevance of the developed technique to the description of neutrino flavor oscillations is examined.
\end{abstract}

PACS numbers: 11.10.-z, 14.60.Pq

Keywords: particle mixing, external fields, neutrino flavor oscillations

\section{INTRODUCTION}

The studying of the evolution of elementary particles systems with mixing is an important problem of high energy physics. Nowadays we have received the experimental confirmation that there exists the mixing in the lepton sector of the standard model (see, e.g., Ref. [1]) which leads to the transitions between different neutrino flavors, i.e. to neutrino flavor oscillations.

Recently considerable efforts were made to theoretically describe the evolution of flavor neutrinos with mixing in order to substantiate the results of the quantum mechanical approach to the problem in question (see Ref. [2, 3] ). The issue of the description of neutrino flavor oscillations with help of field theory methods is important since some problems arise when the quantum mechanics is used to describe neutrino oscillations. For instance, it is not clear (i) whether energies or momenta of different neutrino eigenstates are equal, (ii) whether neutrino oscillations occur in space or in time, (iii) whether it is important to take into account the multicomponent structure of neutrino wave functions etc. Some of the unresolved questions of the neutrino flavor oscillations problem are also briefly outlined in Ref. [4].

It is necessary to mention that in Refs. $[5,6,6,6,8,9$, 10, 11, 12, 13] the quantum field theory was applied for the studying of neutrino flavor oscillations in vacuum. In Ref. [5] the authors studied neutrino oscillations with the most general neutrino mass matrix and derived sum rules in neutrino oscillations. The authors of Refs. [6, 7] discussed neutrino oscillations in a similar way as in Ref. [5].

*Electronic address: dvmaxim@cc.jyu.fi
In those works the evolution of neutrino mass eigenstates was considered, i.e. one formulates neutrino oscillations problem without resort to flavor eigenstates. It should be noticed that, in a sense, the problem of neutrino flavor oscillations is incorrectly formulated because in various processes where a neutrino participates, emitted particles can belong to several neutrino generations. For example, together with muon neutrinos in a pion decay, a small number of electron neutrinos is produced (see also Sec. IV). Hence we should rewrite neutrino emission and detection vertices in terms of mass eigenstates. Then we can examine neutrino emission and detection processes by studying incoming and outgoing charged leptons as in Refs. [5, [6, 7]. These particles are not mixed and thus they can serve as properly defined in and out states.

In Ref. [8] the corrections to the quantum mechanical transition probability formula were obtained when the evolution of Fock states of flavor neutrinos was examined. In Ref. 9] it was demonstrated that neutrino oscillations can be described in terms of entangled flavor states. Several processes involving neutrino weak states, constructed in Ref. [8], were calculated in Ref. [10]. The incompatible results were revealed to appear if one uses the inequivalent vacua model proposed in Ref. 8]. The $S$-matrix approach was used in Ref. 11 to examine the coherence problem in neutrino flavor oscillations. It was shown in Ref. [12] that neutrino oscillations could be suppressed in a gedanken experiment where a neutrino is produced in a crystal by a relativistic electron. Neutrino flavor oscillations were analyzed in Ref. [13] using the path integral method. Finally it should be mentioned that various theoretical approaches to neutrino flavor oscillations were reviewed in Ref. [14] which also contains the comprehensive bibliography.

We demonstrated in Ref. [15] that neutrino flavor oscillations in vacuum, in matter and in electromagnetic fields 
of various configurations could be described in frames of the relativistic quantum mechanics approach. In those works we formulated the initial condition problem and studied the time evolution of neutrino mass eigenstates using Pauli-Jordan function, or its analog taking into account an external field. As a result we received the transition probabilities depending on time. Therefore that method is the most natural generalization, which accounts for the coordinate dependence of neutrinos wave functions, of the quantum mechanical treatment of neutrino oscillations. The weakness of that approach consists in the fact that to obtain a stable oscillations picture one should prepare rather broad, in space, initial wave packet of a flavor neutrino. Such initial conditions are very difficult to implement. In order to overwhelm that difficulty one has to consider more realistic models for neutrino emission processes.

This paper continues the series of our publications on the theory of neutrino flavor oscillations. The purpose of the present work is to provide a deeper understanding of neutrino flavor oscillations in vacuum. We study the evolution of mixed scalar (Sec. III) and spinor (Sec. III) particles emitted by classical sources. We start from Lorentz invariant Lagrangians in $(3+1)$-dimensional space-time. Therefore all the results are valid for arbitrary energies of emitted particles. The fields distributions of scalar and spinor particles exactly accounting for the external sources are obtained. Then for the external fields with the specific time and coordinates dependences we derive the expressions for energy densities, for scalar fields, and intensities, for spinor fields. These quantities are examined in the limit of rapidly oscillating external fields. It is shown that the considered expressions coincide with the common formulae for transition and survival probabilities of neutrino flavor oscillations in vacuum. Moreover in Sec. IIIB we study the evolution of fermion fields with the general mass matrix and examine the appearance of antiparticles. We discuss our results in Sec. IV and suggest that the developed formalism is a theoretical model for the process of neutrino flavor oscillations. The approximations made in deriving of major results are considered in Appendix $\mathrm{A}$.

\section{EVOLUTION OF SCALAR PARTICLES}

In this section we study the dynamics of $N$ complex scalar particles $\varphi=\left(\varphi_{1}, \ldots, \varphi_{N}\right)$ with mixing. The scalar particles in question are supposed to interact with the external classical fields $f_{\lambda}$. The Lagrangian for this system has the following form:

$$
\begin{aligned}
\mathcal{L}(\boldsymbol{\varphi})= & \sum_{\lambda=1}^{N} \partial_{\mu} \varphi_{\lambda}^{\dagger} \partial^{\mu} \varphi_{\lambda}-\sum_{\lambda \lambda^{\prime}=1}^{N} m_{\lambda \lambda^{\prime}}^{2} \varphi_{\lambda}^{\dagger} \varphi_{\lambda^{\prime}} \\
& +\sum_{\lambda=1}^{N}\left(f_{\lambda}^{\dagger} \varphi_{\lambda}+\varphi_{\lambda}^{\dagger} f_{\lambda}\right),
\end{aligned}
$$

where $\left(m_{\lambda \lambda^{\prime}}^{2}\right)$ is mass martix. We suppose that it is real and symmetric. The non-diagonal elements of this matrix are called the vacuum mixing terms. The external sources $f_{\lambda}=f_{\lambda}(\mathbf{r}, t)$ in Eq. (2.1) are taken to be arbitrary functions of coordinates and time.

To describe the evolution of the considered system we make the matrix transformation of the Lagrangian (2.1) and introduce the new set of the scalar fields $u_{a}$,

$$
\varphi_{\lambda}=\sum_{a=1}^{N} U_{\lambda a} u_{a}, \quad \varphi_{\lambda}^{\dagger}=\sum_{a=1}^{N} u_{a}^{\dagger} U_{a \lambda}^{\dagger}
$$

in such a way to diagonalize the matrix $\left(m_{\lambda \lambda^{\prime}}^{2}\right)$, i.e. to eliminate the vacuum mixing terms in Eq. 2.1). It should be noted that the matrix $\left(U_{\lambda a}\right)$ in Eq. (2.2) is orthogonal.

The Lagrangian expressed in terms of the fields $\mathbf{u}=$ $\left(u_{1}, \ldots, u_{N}\right)$ has the following form:

$$
\begin{aligned}
\mathcal{L}(\mathbf{u})= & \sum_{a=1}^{N}\left(\partial_{\mu} u_{a}^{\dagger} \partial^{\mu} u_{a}-m_{a}^{2} u_{a}^{\dagger} u_{a}\right) \\
& +\sum_{a=1}^{N}\left(g_{a}^{\dagger} u_{a}+u_{a}^{\dagger} g_{a}\right)
\end{aligned}
$$

where $m_{a}$ are the masses of the fields $u_{a}$. The new sources $g_{a}$ are obtained with help of Eq. (2.2),

$$
g_{a}=\sum_{\lambda=1}^{N} U_{a \lambda}^{\dagger} f_{\lambda}, \quad g_{a}^{\dagger}=\sum_{\lambda=1}^{N} f_{\lambda}^{\dagger} U_{\lambda a} .
$$

The dynamic equation for the field $u_{a}$ is the inhomogeneous Klein-Gordon equation,

$$
\left(\square-m_{a}^{2}\right) u_{a}=-g_{a},
$$

where $\square=-\partial_{\mu} \partial^{\mu}$ is the d'Alembertian operator. It is worth mentioning that the squares of masses $m_{a}^{2}$ of the fields $u_{a}$ are the eigenvalues of the matrix $\left(m_{\lambda \lambda^{\prime}}^{2}\right)$.

The solution to Eq. (2.5) for the arbitrary function $g_{a}$ has the form (see, e.g., Ref. [16])

$$
u_{a}(\mathbf{r}, t)=\int \mathrm{d}^{3} \mathbf{r}^{\prime} \mathrm{d} t^{\prime} D_{a}^{\mathrm{ret}}\left(\mathbf{r}-\mathbf{r}^{\prime}, t-t^{\prime}\right) g_{a}\left(\mathbf{r}^{\prime}, t^{\prime}\right),
$$

where $D_{a}^{\text {ret }}(\mathbf{r}, t)$ is the retarded Green function. This function obeys the equation,

$$
\begin{aligned}
& \left(\square-m_{a}^{2}\right) D_{a}^{\mathrm{ret}}(x)=-\delta^{4}(x), \\
& D_{a}^{\mathrm{ret}}(x)=0 \quad \text { for } \quad t<0,
\end{aligned}
$$

where $x^{\mu}=(t, \mathbf{r})$. The explicit form of the function $D_{a}^{\text {ret }}(x)$ can be found in Ref. [17],

$$
\begin{aligned}
D_{a}^{\mathrm{ret}}(\mathbf{r}, t) & =\int \frac{\mathrm{d}^{4} p}{(2 \pi)^{4}} \frac{e^{\mathrm{i} p x}}{m_{a}^{2}-p^{2}+\mathrm{i} \epsilon p^{0}} \\
& =\frac{1}{2 \pi} \theta(t)\left\{\delta\left(s^{2}\right)-\theta\left(s^{2}\right) \frac{m_{a}}{2 s} J_{1}\left(m_{a} s\right)\right\},
\end{aligned}
$$


where $\theta(t)$ is the Heaviside step function, $J_{1}(z)$ is the first order Bessel function, $s=\sqrt{t^{2}-r^{2}}$ and $r=|\mathbf{r}|$.

Now it is necessary to specify the time and spatial coordinates dependence of the external fields $f_{\lambda}(\mathbf{r}, t)$. We assume that the sources are localized in space and emit harmonic radiation,

$$
f_{\lambda}(\mathbf{r}, t)=\theta(t) f_{\lambda}^{(0)} e^{-\mathrm{i} E t} \delta^{3}(\mathbf{r}),
$$

where $f_{\lambda}^{(0)}$ is the amplitude of the source and $E$ is its frequency, the analog of the energy of emitted particles. In this case Eq. (2.6) is rewritten in the following way:

$$
u_{a}(\mathbf{r}, t)=g_{a}^{(0)} e^{-\mathrm{i} E t} \int_{0}^{t} \mathrm{~d} \tau D_{a}^{\mathrm{ret}}(\mathbf{r}, \tau) e^{\mathrm{i} E \tau},
$$

where

$$
g_{a}^{(0)}=\sum_{\lambda=1}^{N} U_{a \lambda}^{\dagger} f_{\lambda}^{(0)}
$$

is the time independent component of the function $g_{a}$.

One can see that two major terms appear in Eq. 2.10 [see also Eq. (2.8)] while integrating over $\tau$

$$
\int_{0}^{t} \mathrm{~d} \tau D_{a}^{\mathrm{ret}}(\mathbf{r}, \tau) e^{\mathrm{i} E \tau}=\mathcal{I}_{1}+\mathcal{I}_{2} .
$$

They are

$$
\begin{aligned}
\mathcal{I}_{1} & =\frac{1}{2 \pi} \int_{0}^{t} \mathrm{~d} \tau \delta\left(s^{2}\right) e^{\mathrm{i} E \tau}=\frac{\theta(t-r)}{4 \pi r} e^{\mathrm{i} E r}, \\
\mathcal{I}_{2} & =-\frac{m_{a}}{4 \pi} \int_{0}^{t} \mathrm{~d} \tau \theta\left(s^{2}\right) \frac{J_{1}\left(m_{a} s\right)}{s} e^{\mathrm{i} E \tau} \\
& =-\frac{m_{a}}{4 \pi} \theta(t-r) \int_{0}^{x_{m}} \mathrm{~d} x \frac{J_{1}\left(m_{a} x\right)}{\sqrt{r^{2}+x^{2}}} e^{\mathrm{i} E \sqrt{r^{2}+x^{2}}},
\end{aligned}
$$

where $x_{m}=\sqrt{t^{2}-r^{2}}$ and $s=\sqrt{\tau^{2}-r^{2}}$. It is interesting to notice that both $\mathcal{I}_{1}$ and $\mathcal{I}_{2}$ in Eqs. (2.12) and (2.13) are equal to zero if $t<r$. It means that the initial perturbation from a source placed at the point $\mathbf{r}=0$ reaches a detector placed at the point $\mathbf{r}$ only after the time $t>r$.

Despite the integral $\mathcal{I}_{1}$ in Eq. (2.12) is expressed in terms of the elementary functions, the integral $\mathcal{I}_{2}$ in Eq. (2.13) cannot be computed analytically for arbitrary $r$ and $t$. However some reasonable assumptions can be made to simplify the considered expression. An observer is at the fixed distance from a source. As we have already mentioned one detects a signal starting from $t>r$. It is obvious that a non-stationary rapidly oscillating signal is detected when the wave front just arrives to a detector, i.e. when $t \gtrsim r$. The situation is analogous to waves propagating on the water surface. Therefore, if we suppose that one starts observing particles when the nonstationary signal attenuates, i.e. $t \gg r$ or $x_{m} \rightarrow \infty$, we can avoid relaxation phenomena. In this case the integral $\mathcal{I}_{2}$ can be computed analytically,

$$
\mathcal{I}_{2}=-\frac{1}{4 \pi r}\left(e^{\mathrm{i} E r}-e^{\mathrm{i} p_{a} r}\right)
$$

where $p_{a}=\sqrt{E^{2}-m_{a}^{2}}$ is the analog of the particle momentum.

To obtain Eq. (2.14) we use the known values of the integrals,

$$
\begin{array}{rl}
\int_{0}^{\infty} \mathrm{d} & x \frac{J_{\nu}(m x)}{\sqrt{r^{2}+x^{2}}} \sin \left(E \sqrt{r^{2}+x^{2}}\right) \\
= & \frac{\pi}{2} J_{\nu / 2}\left[\frac{r}{2}\left(E-\sqrt{E^{2}-m^{2}}\right)\right] \\
& \times J_{-\nu / 2}\left[\frac{r}{2}\left(E+\sqrt{E^{2}-m^{2}}\right)\right], \\
\int_{0}^{\infty} \mathrm{d} & \frac{J_{\nu}(m x)}{\sqrt{r^{2}+x^{2}}} \cos \left(E \sqrt{r^{2}+x^{2}}\right) \\
= & -\frac{\pi}{2} J_{\nu / 2}\left[\frac{r}{2}\left(E-\sqrt{E^{2}-m^{2}}\right)\right] \\
& \times N_{-\nu / 2}\left[\frac{r}{2}\left(E+\sqrt{E^{2}-m^{2}}\right)\right],
\end{array}
$$

and the fact that the Bessel and Neumann functions of $\pm 1 / 2$ order

$$
\begin{aligned}
J_{1 / 2}(z)=N_{-1 / 2}(z) & =\sqrt{\frac{2}{\pi z}} \sin z, \\
J_{-1 / 2}(z) & =\sqrt{\frac{2}{\pi z}} \cos z,
\end{aligned}
$$

are expressed in terms of the elementary functions. The approximations made in derivation of Eq. (2.14) are analysed in Appendix A.

Using Eqs. (2.12) and (2.14) we obtain the field distribution of $u_{a}$,

$$
u_{a}(\mathbf{r}, t)=e^{-\mathrm{i} E t+\mathrm{i} p_{a} r} \frac{g_{a}^{(0)}}{4 \pi r} .
$$

Then it is necessary to describe the evolution of the fields $\varphi_{\lambda}$. With help of Eqs. (2.2) and (2.15) one receives the field distribution of $\varphi_{\lambda}$ in the following form:

$$
\varphi_{\lambda}(\mathbf{r}, t)=e^{-\mathrm{i} E t} \sum_{a \lambda^{\prime}=1}^{N} U_{\lambda a} U_{a \lambda^{\prime}}^{\dagger} e^{\mathrm{i} p_{a} r} \frac{f_{\lambda^{\prime}}^{(0)}}{4 \pi r} .
$$

We remind that this formula is valid if $t \gg r$.

To be more elucidative we consider the system of only two scalar fields, $\varphi_{1}$ and $\varphi_{2}$. In this case the matrix $\left(U_{\lambda a}\right)$ in Eq. (2.2) is parametrized with help of the mixing angle $\theta$,

$$
\left(U_{\lambda a}\right)=\left(\begin{array}{cc}
\cos \theta & -\sin \theta \\
\sin \theta & \cos \theta
\end{array}\right) .
$$

We also adopt the following amplitudes of the external sources: $f_{1}^{(0)}=0$ and $f_{2}^{(0)} \equiv f \neq 0$. If we discussed the evolution of flavor neutrinos, that is in fact done in Sec. III such a choice of the external fields would mean that $\varphi_{1}$ would be a muon or a $\tau$-neutrino and $\varphi_{2}-$ an electron neutrino. There would be a source of only electron neutrinos at $\mathbf{r}=0$ and a detector at the distance $r$ from a source. 
Using Eqs. (2.16) and (2.17) we obtain the fields distributions of $\varphi_{1,2}$ in the following form:

$$
\begin{aligned}
& \varphi_{1}(\mathbf{r}, t)=\sin \theta \cos \theta e^{-\mathrm{i} E t} \frac{f}{4 \pi r}\left(e^{\mathrm{i} p_{1} r}-e^{\mathrm{i} p_{2} r}\right), \\
& \varphi_{2}(\mathbf{r}, t)=e^{-\mathrm{i} E t} \frac{f}{4 \pi r}\left(\sin ^{2} \theta e^{\mathrm{i} p_{1} r}+\cos ^{2} \theta e^{\mathrm{i} p_{2} r}\right) .
\end{aligned}
$$

It was demonstrated in Ref. [15] that the measurable quantity for a scalar field is the energy density rather than the field distribution. Constructing the time component $T^{00}$ of the energy momentum tensor of $\varphi_{\lambda}$ we can write down the energy density in the following way:

$$
\begin{aligned}
\mathcal{H}_{\lambda}(\mathbf{r}, t) & =T^{00}\left[\varphi_{\lambda}(\mathbf{r}, t)\right] \\
& =\left|\dot{\varphi}_{\lambda}\right|^{2}+\left|\nabla \varphi_{\lambda}\right|^{2}+m_{\lambda \lambda}^{2}\left|\varphi_{\lambda}\right|^{2}
\end{aligned}
$$

Now let us discuss the high frequency approximation of the obtained results: $E \gg m_{1,2}$. If we again referred to the flavor neutrinos evolution (see Sec. III), it would mean that ultrarelativistic neutrinos would be emitted. Decomposing the "momenta" $p_{a} \approx E-m_{a}^{2} /(2 E)$ in Eqs. (2.18) and taking into account that $\mathcal{H}_{\lambda} \approx\left|\dot{\varphi}_{\lambda}\right|^{2}+$ $\left|\nabla \varphi_{\lambda}\right|^{2} \approx 2 E^{2}\left|\varphi_{\lambda}\right|^{2}$ one receives the expressions for the energy densities

$$
\begin{aligned}
\mathcal{H}_{1}(r)= & 2 E^{2} \frac{|f|^{2}}{(4 \pi r)^{2}} \\
& \times\left\{\sin ^{2}(2 \theta) \sin ^{2}\left(\frac{\Delta m^{2}}{4 E} r\right)+\mathcal{O}\left(\frac{m_{a}^{2}}{E^{2}}\right)\right\}, \\
\mathcal{H}_{2}(r)= & 2 E^{2} \frac{|f|^{2}}{(4 \pi r)^{2}} \\
& \times\left\{1-\sin ^{2}(2 \theta) \sin ^{2}\left(\frac{\Delta m^{2}}{4 E} r\right)+\mathcal{O}\left(\frac{m_{a}^{2}}{E^{2}}\right)\right\},
\end{aligned}
$$

where we use the common notation $\Delta m^{2}=m_{1}^{2}-m_{2}^{2}$.

With help of Eqs. (2.20) we obtain the transition and survival probabilities in the following way:

$$
\begin{aligned}
& P_{2 \rightarrow 1}^{(\text {scalar })}(r) \sim \sin ^{2}(2 \theta) \sin ^{2}\left(\frac{\Delta m^{2}}{4 E} r\right), \\
& P_{2 \rightarrow 2}^{\text {(scalar) }}(r) \sim 1-\sin ^{2}(2 \theta) \sin ^{2}\left(\frac{\Delta m^{2}}{4 E} r\right) .
\end{aligned}
$$

It can be seen that Eqs. (2.21) and (2.22) coincide with the usual formulae for transition and survival probabilities of neutrino flavor oscillations in vacuum that were obtained in frames of the quantum mechanical approach [2, 3].

\section{EVOLUTION OF SPINOR PARTICLES}

In this section we consider the problem analogous to that we discuss in Sec. II namely the evolution of $N$ spinor particles $\boldsymbol{\nu}=\left(\nu_{1}, \ldots, \nu_{N}\right)$ with mixing under the influence of the external classical fields $f_{\lambda}^{\mu}$.
The Lorentz invariant vacuum, i.e. without external fields, Lagrangian generally has the form,

$$
\begin{aligned}
\mathcal{L}(\boldsymbol{\nu})= & \sum_{\lambda=1}^{N}\left(\overline{\nu_{\lambda}^{\mathrm{L}} \mathrm{i}} \gamma^{\mu} \partial_{\mu} \nu_{\lambda}^{\mathrm{L}}+\overline{\nu_{\lambda}^{\mathrm{R}} \mathrm{i}} \gamma^{\mu} \partial_{\mu} \nu_{\lambda}^{\mathrm{R}}\right) \\
& -\sum_{\lambda \lambda^{\prime}=1}^{N}\left(m_{\lambda \lambda^{\prime}}^{\mathrm{D}} \overline{\nu_{\lambda}^{\mathrm{L}}} \nu_{\lambda^{\prime}}^{\mathrm{R}}+m_{\lambda \lambda^{\prime}}^{\mathrm{L}}\left(\nu_{\lambda}^{\mathrm{L}}\right)^{\mathrm{T}} C \nu_{\lambda^{\prime}}^{\mathrm{L}}\right. \\
& \left.+m_{\lambda \lambda^{\prime}}^{\mathrm{R}}\left(\nu_{\lambda}^{\mathrm{R}}\right)^{\mathrm{T}} C \nu_{\lambda^{\prime}}^{\mathrm{R}}+\text { h.c. }\right)
\end{aligned}
$$

where $\nu_{\lambda}^{\mathrm{L}, \mathrm{R}}=(1 / 2)\left(1 \mp \gamma^{5}\right) \nu_{\lambda}$ are the chiral projections, $\left(m_{\lambda \lambda^{\prime}}^{\mathrm{D}}\right),\left(m_{\lambda \lambda^{\prime}}^{\mathrm{L}}\right),\left(m_{\lambda \lambda^{\prime}}^{\mathrm{R}}\right)$ are the Dirac as well as (left and right) Majorana mass matrices and $C=\mathrm{i} \gamma^{2} \gamma^{0}$ is the charge conjugation matrix. These mass matrices should satisfy certain requirements which are discussed in Ref. [3]. The Lagrangian (3.1) is CPT-invariant. In this section we adopt the notations for Dirac matrices as in Ref. [18]. The analogous mass terms are generated in theoretical models based on the type-II seesaw mechanism (see, e.g., Ref. [19]).

We supply the Lagrangian (3.1) with the term describing particles interaction with external sources. Suppose that a particle is involved in the left-handed currents interactions,

$$
\mathcal{L}_{\mathrm{int}}=\sum_{\lambda=1}^{N}\left(\bar{\ell}_{\lambda} \gamma_{\mu}^{\mathrm{L}} \nu_{\lambda} f_{\lambda}^{\dagger \mu}+\bar{\nu}_{\lambda} \gamma_{\mu}^{\mathrm{L}} \ell_{\lambda} f_{\lambda}^{\mu}\right)
$$

where $\gamma_{\mu}^{\mathrm{L}}=\gamma_{\mu}\left(1-\gamma^{5}\right) / 2$. The external fields $f_{\lambda}^{\mu}=$ $f_{\lambda}^{\mu}(\mathbf{r}, t)$ are supposed to be arbitrary functions. The physical analog of the Lagrangian (3.2) is the system of flavor neutrinos $\nu_{\lambda}$ interacting with matter by means of the electroweak interactions. In this case $\ell_{\lambda}$ is the charged $\mathrm{SU}(2)$ isodoublet partner of $\nu_{\lambda}$. If we study the neutrino emission in a process like the inverse $\beta$-decay: $p+e^{-} \rightarrow \nu_{e}+n$, the external fields in Eq. (3.2) are (see, e.g., Ref. [20])

$$
\begin{aligned}
& f_{\nu_{e}}^{\mu}=-\sqrt{2} G_{F} \bar{\Psi}_{n} \gamma^{\mu}\left(1-\alpha \gamma^{5}\right) \Psi_{p} \\
& f_{\nu_{\mu}}^{\mu}=0, \quad f_{\nu_{\tau}}^{\mu}=0
\end{aligned}
$$

where $\Psi_{p}$ and $\Psi_{n}$ are the wave functions of a proton and a neutron, $\alpha \approx 1.25$ and $G_{\mathrm{F}}$ is the Fermi constant. In Eq. (3.3) we assume that a source consists of electrons, protons and neutrons.

After the diagonalization of the whole mass term of the Lagrangian (3.1) one obtains $2 N$ Majorana particles with different masses [3]. In the following we discuss the case when only Dirac mass term is presented. Then we study the general situation.

\section{A. Dirac mass term}

In this section we suppose that only Dirac mass matrix is non-zero in Eq. (3.1). Analogously to the discussion 
in Sec. II we introduce the new set of spinor fields $\boldsymbol{\psi}=$ $\left(\psi_{1}, \ldots, \psi_{N}\right)$ by means of the matrix transformation. In our situation the mixing matrix is the $N \times N$ unitary matrix $\left(U_{\lambda a}\right)$,

$$
\nu_{\lambda}=\sum_{a=1}^{N} U_{\lambda a} \psi_{a}, \quad \bar{\nu}_{\lambda}=\sum_{a=1}^{N} \bar{\psi}_{a} U_{a \lambda}^{\dagger} .
$$

The corresponding mass eigenstates $\psi_{a}$ are Dirac particles.

When we transform the sum of the Lagrangians (3.1) and (3.2) using Eq. (3.4), it is rewritten in the following way:

$$
\begin{aligned}
\mathcal{L}(\boldsymbol{\psi})= & \sum_{a=1}^{N} \bar{\psi}_{a}\left(\mathrm{i} \gamma^{\mu} \partial_{\mu}-m_{a}\right) \psi_{a} \\
& +\sum_{a=1}^{N}\left(\bar{\Psi}_{a} \psi_{a}+\bar{\psi}_{a} \Psi_{a}\right),
\end{aligned}
$$

where $\Psi_{a}$ is the external source for the fermion $\psi_{a}$,

$$
\Psi_{a}=\sum_{\lambda=1}^{N} U_{a \lambda}^{\dagger} \gamma_{\mu}^{\mathrm{L}} \ell_{\lambda} f_{\lambda}^{\mu}, \quad \bar{\Psi}_{a}=\sum_{\lambda=1}^{N} \bar{\ell}_{\lambda} \gamma_{\mu}^{\mathrm{L}} f_{\lambda}^{\dagger \mu} U_{\lambda a} .
$$

Using Eqs. (3.5) and (3.6) we receive the inhomogeneous Dirac equation for the fermion $\psi_{a}$,

$$
\left(\mathrm{i} \gamma^{\mu} \partial_{\mu}-m_{a}\right) \psi_{a}=-\Psi_{a} .
$$

We mention that the masses $m_{a}$ are the eigenvalues of the matrix $\left(m_{\lambda \lambda^{\prime}}^{\mathrm{D}}\right)$.

The solution to Eq. (3.6) for the arbitrary spinor $\Psi_{a}$ is expressed with help of the retarded Green function for a spinor field (see again Ref. [16]),

$$
\psi_{a}(\mathbf{r}, t)=\int \mathrm{d}^{3} \mathbf{r}^{\prime} \mathrm{d} t^{\prime} S_{a}^{\mathrm{ret}}\left(\mathbf{r}-\mathbf{r}^{\prime}, t-t^{\prime}\right) \Psi_{a}\left(\mathbf{r}^{\prime}, t^{\prime}\right) .
$$

The explicit form of $S_{a}^{\text {ret }}(\mathbf{r}, t)$ can be also found in Ref. [16],

$$
S_{a}^{\mathrm{ret}}(\mathbf{r}, t)=\left(\mathrm{i} \gamma^{\mu} \partial_{\mu}+m_{a}\right) D_{a}^{\mathrm{ret}}(\mathbf{r}, t)
$$

where the function $D_{a}^{\text {ret }}(\mathbf{r}, t)$ is given in Sec. II

To proceed in further calculations it is necessary to define the behavior of the external sources. Let us suppose that the external fields depend on time and spatial coordinates in the similar way as in Sec. II namely

$$
\ell_{\lambda}(\mathbf{r}, t) f_{\lambda}^{\mu}(\mathbf{r}, t)=\theta(t) l_{\lambda} f_{\lambda}^{(0) \mu} e^{-\mathrm{i} E t} \delta^{3}(\mathbf{r}),
$$

where $l_{\lambda}=\ell_{\lambda}^{(0)}$ is the time independent component of the spinor $\ell_{\lambda}$ and $f_{\lambda}^{(0) \mu}$ is the amplitude of the function $f_{\lambda}^{\mu}(\mathbf{r}, t)$. Using Eqs. (3.6) and (3.10) we obtain for $\Psi_{a}$,

$$
\begin{aligned}
\Psi_{a}(\mathbf{r}, t) & =\theta(t) \Psi_{a}^{(0)} e^{-\mathrm{i} E t} \delta^{3}(\mathbf{r}), \\
\Psi_{a}^{(0)} & =\sum_{\lambda=1}^{N} U_{a \lambda}^{\dagger} \gamma_{\mu}^{\mathrm{L}} l_{\lambda} f_{\lambda}^{(0) \mu} .
\end{aligned}
$$

With help of Eqs. (3.9)-3.11) we can rewrite Eq. (3.8) in the following way:

$$
\begin{aligned}
\psi_{a}(\mathbf{r}, t)= & \left(\mathrm{i} \gamma^{\mu} \frac{\partial}{\partial x^{\mu}}+m_{a}\right) \\
& \times\left\{e^{-\mathrm{i} E t} \int_{0}^{t} \mathrm{~d} \tau D_{a}^{\mathrm{ret}}(\mathbf{r}, \tau) e^{\mathrm{i} E \tau}\right\} \Psi_{a}^{(0)} .
\end{aligned}
$$

Now it is possible to use the technique developed in Sec. II. Finally we obtain the field distribution of the fermion $\psi_{a}$,

$$
\psi_{a}(\mathbf{r}, t)=e^{-\mathrm{i} E t+\mathrm{i} p_{a} r} O_{a} \frac{\Psi_{a}^{(0)}}{4 \pi r}
$$

where $O_{a}=\gamma^{0} E-(\gamma \mathbf{n}) p_{a}+m_{a}$ and $\mathbf{n}$ is the unit vector towards a detector. It should be noted that in deriving Eq. (3.13) we differentiate only exponential rather than the factor $1 / r$ because the derivative of $1 / r$ is proportional to $1 / r^{2}$. Such a term is negligible at large distances from a source. We also remind that Eq. (3.13) is valid for $t \gg r$.

Let us turn to the description of the evolution of the fields $\nu_{\lambda}$. Using Eqs. (3.4) and (3.13), we obtain the corresponding field distribution,

$$
\nu_{\lambda}(\mathbf{r}, t)=e^{-\mathrm{i} E t} \sum_{a \lambda^{\prime}=1}^{N} U_{\lambda a} U_{a \lambda^{\prime}}^{\dagger} e^{\mathrm{i} p_{a} r} O_{a} \gamma_{\mu}^{\mathrm{L}} l_{\lambda^{\prime}} \frac{f_{\lambda^{\prime}}^{(0) \mu}}{4 \pi r}
$$

Analogously to Sec. II we study the evolution of only two fermions. The mixing matrix is given in Eq. (2.17). We also choose the amplitudes of the sources in the following way: $f_{1}^{(0) \mu}=0, f_{2}^{(0) \mu} \equiv f^{\mu} \neq 0$ and $l_{2} \equiv l$. The reason for such a choice is similar to that in Sec. II If we suppose that $\nu_{1}$ corresponds to a muon or a $\tau$-neutrino and $\nu_{2}-$ to an electron neutrino, then it means that there is a source of only electron neutrinos and we observe particles at the distance $r$ from a source. Using Eqs. (2.17) and (3.14) we obtain the fields distributions of each of the particles $\nu_{1,2}$,

$$
\begin{aligned}
\nu_{1}(\mathbf{r}, t)= & \sin \theta \cos \theta e^{-\mathrm{i} E t} \frac{f^{\mu}}{4 \pi r} \\
& \times\left(e^{\mathrm{i} p_{1} r} O_{1}-e^{\mathrm{i} p_{2} r} O_{2}\right) \gamma_{\mu}^{\mathrm{L}} l, \\
\nu_{2}(\mathbf{r}, t)= & e^{-\mathrm{i} E t} \frac{f^{\mu}}{4 \pi r} \\
& \times\left(\sin ^{2} \theta e^{\mathrm{i} p_{1} r} O_{1}+\cos ^{2} \theta e^{\mathrm{i} p_{2} r} O_{2}\right) \gamma_{\mu}^{\mathrm{L}} l .
\end{aligned}
$$

In the following we discuss the high frequency approximation: $E \gg m_{1,2}$. It again corresponds to the emission of ultrarelativistic neutrinos.

It was shown in our previous works [15] that the measurable quantity for a spinor particle is the intensity which is defined by the following expression: $I_{\lambda}(\mathbf{r}, t)=$ $\left|\nu_{\lambda}(\mathbf{r}, t)\right|^{2}$. With help of Eqs. (3.15) we get the intensities 
in the form

$$
\begin{aligned}
I_{1}(r)= & -2 E^{2} \frac{f^{\dagger \mu} f^{\nu}}{(4 \pi r)^{2}}\left\langle T_{\mu \nu}\right\rangle \\
& \times\left\{\sin ^{2}(2 \theta) \sin ^{2}\left(\frac{\Delta m^{2}}{4 E} r\right)+\mathcal{O}\left(\frac{m_{a}^{2}}{E^{2}}\right)\right\}, \\
I_{2}(r)= & -2 E^{2} \frac{f^{\dagger \mu} f^{\nu}}{(4 \pi r)^{2}}\left\langle T_{\mu \nu}\right\rangle \\
& \times\left\{1-\sin ^{2}(2 \theta) \sin ^{2}\left(\frac{\Delta m^{2}}{4 E} r\right)+\mathcal{O}\left(\frac{m_{a}^{2}}{E^{2}}\right)\right\},
\end{aligned}
$$

where $\left\langle T_{\mu \nu}\right\rangle=l^{\dagger} \gamma_{\mu}^{\dagger}(\boldsymbol{\alpha} \mathbf{n}) \gamma_{\nu} l$ and $\boldsymbol{\alpha}=\gamma^{0} \boldsymbol{\gamma}$. It is possible to calculate the components of the tensor $\left\langle T_{\mu \nu}\right\rangle$. They depend on the properties of the fermion $\ell_{2} \equiv \ell$,

$$
\begin{gathered}
\left\langle T_{00}\right\rangle=-(\mathbf{v n}), \quad\left\langle T_{0 i}\right\rangle=\left\langle T_{i 0}\right\rangle^{*}=n_{i}-\mathrm{i}[\mathbf{n} \times \boldsymbol{\zeta}]_{i}, \\
\left\langle T_{i j}\right\rangle=\delta_{i j}(\mathbf{v n})-\left(v_{i} n_{j}+v_{j} n_{i}\right)+\mathrm{i} \varepsilon_{i j k} n_{k},
\end{gathered}
$$

where $\mathbf{v}=\langle\boldsymbol{\alpha}\rangle$ is the velocity of fermion $\ell, \boldsymbol{\zeta}=\langle\boldsymbol{\Sigma}\rangle$ is its spin and $\boldsymbol{\Sigma}=\gamma^{5} \boldsymbol{\alpha}$. It should be noted that, if we study the evolution of flavor neutrinos and $\nu_{2}$ corresponds to an electron neutrino, the fermion $\ell$ is an electron.

Let us discuss the simplified case when the spatial components of the four-vector $f^{\mu}$ are equal to zero: $\mathbf{f}=0$. It corresponds to the neutrino emission by a non-moving and unpolarized source. Using Eq. (3.17) we can rewrite Eq. (3.16) in the following way:

$$
\begin{aligned}
I_{1}(r) \approx & 2 E^{2}(\mathbf{v n}) \frac{\left|f^{0}\right|^{2}}{(4 \pi r)^{2}} \sin ^{2}(2 \theta) \sin ^{2}\left(\frac{\Delta m^{2}}{4 E} r\right), \\
I_{2}(r) \approx & 2 E^{2}(\mathbf{v n}) \frac{\left|f^{0}\right|^{2}}{(4 \pi r)^{2}} \\
& \times\left\{1-\sin ^{2}(2 \theta) \sin ^{2}\left(\frac{\Delta m^{2}}{4 E} r\right)\right\} .
\end{aligned}
$$

Now we are able to introduce the transition and survival probabilities on the basis of Eq. (3.18),

$$
\begin{aligned}
& P_{2 \rightarrow 1}^{(\text {spinor })}(r) \sim \sin ^{2}(2 \theta) \sin ^{2}\left(\frac{\Delta m^{2}}{4 E} r\right), \\
& P_{2 \rightarrow 2}^{\text {(spinor) }}(r) \sim 1-\sin ^{2}(2 \theta) \sin ^{2}\left(\frac{\Delta m^{2}}{4 E} r\right) .
\end{aligned}
$$

It should be noted that Eqs. (3.19) and (3.20) are the same as the common formulae for the description of neutrino flavor oscillations in vacuum [2, 3]. These expressions also coincide with Eqs. (2.21) and (2.22) derived for the case of the mixed scalar fields.

\section{B. General mass term}

To begin analyzing the dynamics of mixed fermions with the general mass term (3.1) we rewrite the interaction Lagrangian (3.2) in the equivalent form,

$$
\mathcal{L}_{\text {int }}=\sum_{\lambda=1}^{N}\left(\overline{\ell_{\lambda}^{\mathrm{L}}} \gamma_{\mu} \nu_{\lambda}^{\mathrm{L}} f_{\lambda}^{\dagger \mu}+\overline{\nu_{\lambda}^{\mathrm{L}}} \gamma_{\mu} \ell_{\lambda}^{\mathrm{L}} f_{\lambda}^{\mu}\right) .
$$

Now we can introduce mass eigenstates $\psi_{a}$. The analog of Eq. (3.4) is (see also Ref. [3])

$$
\nu_{\lambda}^{\mathrm{L}}=\sum_{a=1}^{2 N} U_{\lambda a} \psi_{a}^{\mathrm{L}}, \quad \overline{\nu_{\lambda}^{\mathrm{L}}}=\sum_{a=1}^{2 N} \overline{\psi_{a}^{\mathrm{L}}} U_{a \lambda}^{\dagger} .
$$

where the matrix $\left(U_{\lambda a}\right)$ is the rectangular $N \times 2 N$ matrix. Note that it is not unitary. We also mention that the mass eigenstates $\psi_{a}$ are Majorana particles. To implement the complete diagonalization of the mass term (3.1) it is necessary to use $2 N \times 2 N$ unitary matrix, i.e. along with Eq. (3.22) one should consider also the transformation of the right-handed chiral components of the particles $\nu_{\lambda}$

$$
\nu_{\lambda}^{\mathrm{R}}=\sum_{a=1}^{2 N} V_{\lambda a} \psi_{a}^{\mathrm{R}} .
$$

Note that the matrices $\left(U_{\lambda a}\right)$ and $\left(V_{\lambda a}\right)$ in Eqs. (3.22) and (3.23) are independent. The modern parameterization for these matrices is given in Ref. [21].

The Lagrangian for the particles $\psi_{a}$ takes the form,

$$
\begin{aligned}
\mathcal{L}(\boldsymbol{\psi})= & \sum_{a=1}^{2 N} \overline{\psi_{a}^{\mathrm{L}} \mathrm{i}} \gamma^{\mu} \partial_{\mu} \psi_{a}^{\mathrm{L}} \\
& -\sum_{a=1}^{2 N}\left(m_{a} \overline{\psi_{a}^{\mathrm{L}}} \psi_{a}^{\mathrm{R}}-\overline{\Psi_{a}^{\mathrm{R}}} \psi_{a}^{\mathrm{L}}+\text { h.c. }\right),
\end{aligned}
$$

where the external sources $\Psi_{a} \equiv \Psi_{a}^{\mathrm{R}}$ have the same form as in Eq. (3.6).

Note that Majorana spinors are equivalent to twocomponent Weil spinors [23]. Hence we can rewrite the spinors $\psi_{a}^{\mathrm{L}, \mathrm{R}}$ and $\Psi_{a}$ as

$$
\psi_{a}^{\mathrm{L}}=\left(\begin{array}{c}
0 \\
\eta_{a}
\end{array}\right), \quad \psi_{a}^{\mathrm{R}}=\left(\begin{array}{c}
\mathrm{i} \sigma_{2} \eta_{a}^{*} \\
0
\end{array}\right), \quad \Psi_{a}=\left(\begin{array}{c}
\phi_{a} \\
0
\end{array}\right),
$$

where

$$
\phi_{a}=\sum_{\lambda=1}^{N} U_{a \lambda}^{\dagger} \chi_{\lambda} f_{\lambda}^{0} .
$$

To obtain Eq. (3.26) we suppose as in Sec. IIIA that the vectors $f_{\lambda}^{\mu}$ have only time component $f_{\lambda}^{0}$. We also assume that $\left(\ell_{\lambda}^{\mathrm{L}}\right)^{\mathrm{T}}=\left(0, \chi_{\lambda}\right)$. To derive Eq. (3.26) it is crucial that only left-handed currents interactions are presented in Eqs. (3.2) and (3.6).

It is useful to rewrite the Lagrangian (3.24) in terms of the two-component spinors $\boldsymbol{\eta}=\left(\eta_{1}, \ldots, \eta_{2 N}\right), \boldsymbol{\eta}^{*}=$ $\left(\eta_{1}^{*}, \ldots, \eta_{2 N}^{*}\right)$ and $\left(\phi_{1}, \ldots, \phi_{2 N}\right)$ [22],

$$
\begin{aligned}
\mathcal{L}\left(\boldsymbol{\eta}, \boldsymbol{\eta}^{*}\right)= & \sum_{a=1}^{2 N} \mathrm{i} \eta_{a}^{\dagger}\left(\partial_{t}-\boldsymbol{\sigma} \boldsymbol{\nabla}\right) \eta_{a} \\
& +\sum_{a=1}^{2 N}\left(\frac{\mathrm{i}}{2} m_{a} \eta_{a}^{\dagger} \sigma_{2} \eta_{a}^{*}+\eta_{a}^{\dagger} \phi_{a}+\text { h.c. }\right) .
\end{aligned}
$$


Using Eq. (3.27) we can receive the wave equation for two-component spinors,

$$
\left(\frac{\partial}{\partial t}-\boldsymbol{\sigma} \nabla\right) \eta_{a}+m_{a} \sigma_{2} \eta_{a}^{*}=\mathrm{i} \phi_{a}
$$

The solutions to Eq. (3.28) have the form (see, e.g., Ref. [22]),

$$
\begin{aligned}
& \eta_{a}(\mathbf{r}, t)=\int \mathrm{d}^{3} \mathbf{r}^{\prime} \mathrm{d} t^{\prime} \mathcal{S}_{a}^{\mathrm{ret}}\left(\mathbf{r}-\mathbf{r}^{\prime}, t-t^{\prime}\right) \phi_{a}\left(\mathbf{r}^{\prime}, t^{\prime}\right), \\
& \eta_{a}^{*}(\mathbf{r}, t)=\int \mathrm{d}^{3} \mathbf{r}^{\prime} \mathrm{d} t^{\prime} \mathcal{R}_{a}^{\mathrm{ret}}\left(\mathbf{r}-\mathbf{r}^{\prime}, t-t^{\prime}\right) \phi_{a}\left(\mathbf{r}^{\prime}, t^{\prime}\right),
\end{aligned}
$$

where the retarded Green functions are expressed as (see also Ref. [22]),

$$
\begin{aligned}
\mathcal{S}_{a}^{\mathrm{ret}}(\mathbf{r}, t) & =\mathrm{i} \tilde{\sigma}^{\mu} \partial_{\mu} D_{a}^{\mathrm{ret}}(\mathbf{r}, t), \\
\mathcal{R}_{a}^{\mathrm{ret}}(\mathbf{r}, t) & =\mathrm{i} m_{a} \sigma_{2} D_{a}^{\mathrm{ret}}(\mathbf{r}, t) .
\end{aligned}
$$

Here $\partial_{\mu}=\left(\partial_{t}, \boldsymbol{\nabla}\right), \tilde{\sigma}^{\mu}=(\widehat{\mathbb{1}}, \boldsymbol{\sigma}), \widehat{\mathbb{1}}$ is the $2 \times 2$ unit matrix and $D_{a}^{\text {ret }}(\mathbf{r}, t)$ is given in Eq. (2.8). One can check that Eq. (3.29) and (3.30), along with the definition of the retarded Green functions (3.31), represent the solutions to Eq. (3.28) by means of direct substituting and using Eq. (2.7).

Let us assume that the sources $\phi_{a}$ depend on time and spatial coordinates as in previous sections,

$$
\begin{aligned}
\phi_{a}(\mathbf{r}, t) & =\theta(t) \phi_{a}^{(0)} e^{-\mathrm{i} E t} \delta^{3}(\mathbf{r}), \\
\phi_{a}^{(0)} & =\sum_{\lambda=1}^{N} U_{a \lambda}^{\dagger} f_{\lambda} \chi_{\lambda}^{(0)},
\end{aligned}
$$

where $\chi_{\lambda}^{(0)}$ is the time independent component of $\chi_{\lambda}$. In deriving of Eq. (3.32) from Eq. (3.26) we introduce the new quantities $f_{\lambda} \equiv f_{\lambda}^{(0) 0}$, where $f_{\lambda}^{(0) 0}$ is the time independent part of $f_{\lambda}^{0}$, to simplify the notations.

On the basis of Eqs. (3.29)-(3.32) and using the technique of the previous sections we get the particles wave functions,

$$
\begin{aligned}
& \eta_{a}(\mathbf{r}, t)=\mathrm{i} \tilde{\sigma}^{\mu} \partial_{\mu}\left[e^{-\mathrm{i} E t+\mathrm{i} p_{a} r}\right] \frac{\phi_{a}^{(0)}}{4 \pi r}, \\
& \eta_{a}^{*}(\mathbf{r}, t)=\mathrm{i} m_{a} \sigma_{2} e^{-\mathrm{i} E t+\mathrm{i} p_{a} r} \frac{\phi_{a}^{(0)}}{4 \pi r} .
\end{aligned}
$$

To derive Eqs. (3.33) and (3.34) we suppose that $t \gg r$. The derivatives in Eq. (3.33) are applied on the exponent only because of the same reasons as in Sec. IIIA.

Using Eqs. (3.22), (3.33) and (3.34) as well as the following identity:

$$
\begin{aligned}
& \left(\nu_{\lambda}^{\mathrm{L}}\right)^{c}=\left(\nu_{\lambda}^{c}\right)^{\mathrm{R}}=\sum_{a=1}^{2 N} U_{\lambda a}^{*} \psi_{a}^{\mathrm{R}}, \\
& \left(\nu_{\lambda}^{\mathrm{L}}\right)^{c}=C\left(\overline{\nu_{\lambda}^{\mathrm{L}}}\right)^{\mathrm{T}},
\end{aligned}
$$

we get the wave functions of $\nu_{\lambda}^{\mathrm{L}}$ and $\left(\nu_{\lambda}^{\mathrm{L}}\right)^{c}$ as

$$
\begin{aligned}
\nu_{\lambda}^{\mathrm{L}}(\mathbf{r}, t)= & \frac{2 E}{4 \pi r} e^{-\mathrm{i} E t} \sum_{a=1}^{2 N} \sum_{\lambda^{\prime}=1}^{N} e^{\mathrm{i} p_{a} r} U_{\lambda a} U_{a \lambda^{\prime}}^{\dagger} \\
& \times f_{\lambda^{\prime}}\left(\begin{array}{c}
0 \\
\tilde{\chi}_{\lambda^{\prime}}^{(0)}
\end{array}\right) \\
\left(\nu_{\lambda}^{\mathrm{L}}\right)^{c}(\mathbf{r}, t)= & -\frac{2 E}{4 \pi r} e^{-\mathrm{i} E t} \sum_{a=1}^{2 N} \sum_{\lambda^{\prime}=1}^{N} \frac{m_{a}}{2 E} e^{\mathrm{i} p_{a} r} U_{\lambda a}^{*} U_{a \lambda^{\prime}}^{\dagger} \\
& \times f_{\lambda^{\prime}}\left(\begin{array}{c}
\chi_{\lambda^{\prime}}^{(0)} \\
0
\end{array}\right)
\end{aligned}
$$

where $\tilde{\chi}_{\lambda^{\prime}}^{(0)}=(1 / 2)[1-(\boldsymbol{\sigma} \mathbf{n})] \chi_{\lambda^{\prime}}^{(0)}$. In deriving of Eq. (3.36) we suppose that

$$
\left[E-p_{a}(\boldsymbol{\sigma} \mathbf{n})\right] \approx E[1-(\boldsymbol{\sigma} \mathbf{n})],
$$

that is valid for relativistic neutrinos.

It is possible to construct two four-component Majorana spinors from two-component Weil spinors,

$$
\psi_{a}^{(1)}=\left(\begin{array}{c}
\mathrm{i} \sigma_{2}\left(\eta_{a}\right)^{*} \\
\eta_{a}
\end{array}\right), \quad \psi_{a}^{(2)}=\left(\begin{array}{c}
\mathrm{i} \sigma_{2} \eta_{a}^{*} \\
\left(\eta_{a}^{*}\right)^{*}
\end{array}\right)
$$

where $\eta_{a}$ and $\eta_{a}^{*}$ are defined in Eqs. (3.33) and (3.34). One can see that these spinors satisfy the Majorana condition $\left[\psi_{a}^{(1,2)}\right]^{c}=\psi_{a}^{(1,2)}$. We use the spinor $\psi_{a}^{(1)}$ to receive Eq. (3.36) and $\psi_{a}^{(2)}-$ Eq. (3.37). In this case we get that $\nu_{\lambda}^{\mathrm{L}}$ and $\left(\nu_{\lambda}^{\mathrm{L}}\right)^{c}$ are obtained as a result of the evolution of particles emitted from the same source.

It should be mentioned that both $\nu_{\lambda}^{\mathrm{L}}$ and $\left(\nu_{\lambda}^{\mathrm{L}}\right)^{c}$ in Eqs. (3.36) and (3.37) propagate forward in time. To explain this fact let us discuss the complex conjugated equation (3.29). Performing the same computations one arrives to the analog of Eq. (3.37) which would depend on time as $e^{\mathrm{i} E t}$. However this wave function describes a particle emitted by the source different from that discussed here. Indeed, if we studied the complex conjugated Eq. (3.29), the integrand there would be $\mathcal{S}_{a}^{\text {ret* }} \phi_{a}^{*}$. It would mean that the source in Eq. (3.32) would be proportional to $\phi_{a}^{(0) *} e^{\mathrm{i} E t}$, that, in its turn, would signify that $\left(\nu_{\lambda}^{\mathrm{L}}\right)^{c}$ would be emitted in a process involving a lepton which is a charge conjugated counterpart to that discussed here.

Let us illustrate this problem on more physical example. Suppose that we study a neutrino emission in a process like the inverse $\beta$-decay,

$$
p+\ell_{\lambda}^{-} \rightarrow n+\nu_{\lambda} \Rightarrow n+\sum_{a=1}^{2 N} U_{\lambda a} \psi_{a}^{\mathrm{L}}
$$

where $p, n$ and $\ell_{\lambda}^{-}$stand for a proton, a neutron and for a negatively charged lepton. The complex conjugated 
Eq. (3.29) would correspond to a process,

$$
\begin{aligned}
n+\ell_{\lambda}^{+} \rightarrow p+\tilde{\nu}_{\lambda} & \Rightarrow p+\sum_{a=1}^{2 N} U_{\lambda a}^{*}\left(\psi_{a}^{\mathrm{L}}\right)^{c} \\
& \Rightarrow p+\sum_{a=1}^{2 N} U_{\lambda a}^{*} \psi_{a}^{\mathrm{R}},
\end{aligned}
$$

where $\tilde{\nu}_{\lambda}$ and $\ell_{\lambda}^{+}$denote an antineutrino and a positively charged lepton, which is the charge conjugated counterpart to $\ell_{\lambda}^{-}$. In Eq. (3.40) we use the facts that only left-handed interactions exist in nature and the fields $\psi_{a}$ describe Majorana particles. As one can see, Eqs. (3.39) and (3.40) represent two different processes. Hence, if we used $\left(\eta_{a}\right)^{*}$ to obtain $\left(\nu_{\lambda}^{\mathrm{L}}\right)^{c}(\mathbf{r}, t)$, i.e. after a beam of neutrinos passes some distance $r$, it would correspond to the initial reaction (3.40) rather than (3.39). Note that the same result also follows from Eq. (3.38) if we replace $\psi_{a}^{(1)} \leftrightarrow \psi_{a}^{(2)}$ there.

Let us suppose for simplicity that the momentum of the fermion $\ell_{\lambda}$ is parallel to the neutrino momentum. It takes place if a relativistic incoming lepton is studied. We also assume that this fermion is in a state with the definite helicity,

$$
\frac{1}{2}[1-(\boldsymbol{\sigma} \mathbf{n})] \chi_{\lambda}^{(0)}=\chi_{\lambda}^{(0)} .
$$

This expression is again natural for the relativistic fermion $\ell_{\lambda}$. One can notice that interaction Lagrangian (3.21) is written in terms of the left-handed chiral projections of $\ell_{\lambda}$. Therefore, if we study a relativistic lepton, it will have its spin directed oppositely to the particle momentum as one can see from Eq. (3.41).

Using Eqs. (3.36), (3.37) and (3.41) as well as the orthonormality of the two-component spinors $\chi_{\lambda}^{(0)}$, $\left(\chi_{\lambda}^{(0) \dagger} \chi_{\lambda^{\prime}}^{(0)}\right)=\delta_{\lambda \lambda^{\prime}}$, we get the probabilities to detect $\nu_{\lambda}^{\mathrm{L}}$ and $\left(\nu_{\lambda}^{\mathrm{L}}\right)^{c}$ as

$$
\begin{aligned}
P_{\nu_{\lambda}^{\mathrm{L}}}(r) \sim & \sum_{a b=1}^{2 N} \sum_{\lambda^{\prime}=1}^{N} e^{\mathrm{i}\left(p_{a}-p_{b}\right) r} \\
& \times U_{\lambda a} U_{a \lambda^{\prime}}^{\dagger} U_{b \lambda^{\prime}}^{\dagger} U_{\lambda^{\prime} b}\left|f_{\lambda^{\prime}}\right|^{2}, \\
P_{\left(\nu_{\lambda}^{\mathrm{L}}\right)^{c}}(r) \sim & \sum_{a b=1}^{2 N} \sum_{\lambda^{\prime}=1}^{N} \frac{m_{a} m_{b}}{(2 E)^{2}} e^{\mathrm{i}\left(p_{a}-p_{b}\right) r} \\
& \times U_{\lambda a}^{*} U_{a \lambda^{\prime}}^{\dagger} U_{b \lambda^{\prime}}^{\mathrm{T}} U_{\lambda^{\prime} b}\left|f_{\lambda^{\prime}}\right|^{2} .
\end{aligned}
$$

In Eqs. (3.42) and (3.43) we drop the factor $(2 E)^{2} /(4 \pi r)^{2}$.

We can see that Eqs. (3.42) and (3.43) contain the oscillating exponent. This our result reproduces the usual formulae for neutrino oscillations in vacuum [3]. It should be also noticed that the expressions for the probabilities depend on $r$ rather than on $t$ in contrast to our previous works [15]. Note that the problem whether neutrino oscillations happen in space or in time was also discussed in Ref. 24]. The similar coordinate dependence of the probabilities was obtained in Refs. [5, 6, 7] where the problem of neutrino oscillations in vacuum was studied.

It was mentioned in Ref. [5] that oscillations between active and sterile neutrinos are possible in case of the non-unitary matrix $\left(U_{\lambda a}\right)$, i.e. the presence of only Majorana mass terms is not sufficient for the existence of this kind of transitions. The situation is analogous to that considered in the pioneering work [25] where oscillations between neutrinos and antineutrinos were studied. In Eq. (3.43) we obtain that the probability to detect $\left(\nu_{\lambda}^{\mathrm{L}}\right)^{c}$ is suppressed by the factor $m_{a} m_{b} / E^{2}$. It was also mentioned in Refs. [5, 26].

\section{DISCUSSION AND CONCLUSIONS}

In conclusion we mention that we studied the evolution of the systems of mixed particles emitted by classical sources. The cases of both scalar and spinor particles were considered. The fields distributions of particles were found for the arbitrary sources dependences on time and spatial coordinates. Note that the expressions for the fields distributions were received directly from the Lorentz invariant wave equations and thus they are valid for arbitrary energies of emitted particles. It should be also mentioned that all the calculations were carried out in $(3+1)$ dimensions.

We demonstrated that the expressions for energy densities, in the scalar fields case, and intensities, in the spinor fields case, coincide with the usual transition and survival probabilities of neutrino flavor oscillations in vacuum derived within the quantum mechanical approach. Since we study the process of neutrino oscillations on the basis of the field theory method we reproduce not only the quantum mechanical probabilities as the leading terms, but also one can derive the corrections to these expressions. It is known (see Refs. [8, 15]) that the Pontecorvo formula for neutrino oscillations has the corrections $\sim\left(m_{a} / E\right)^{2}$, that are small for ultrarelativistic neutrinos. In the present work we showed that the analogous terms also appear in the expressions for probabilities [see, e.g., Eqs. (2.20) and (3.16)]. In addition, in Sec. IIIB we considered the possibility of the appearance of antiparticles in the initial particles beam.

In our previous works [15] we studied neutrino flavor oscillations in vacuum and in various external fields within the field theoretical approach. In those papers we solved the initial condition problem for the wave equations describing the evolution of neutrino mass eigenstates. To study the dynamics of the particles one should use Pauli-Jordan function as the Green function for the considered initial condition problem. As a result we received the final wave functions corresponding to the time $t$ of the system evolution. It was shown that the corresponding probabilities to detect a flavor neutrino coincide with usual probabilities of neutrino oscillations derived in the Schrödinger equation approach. Therefore 
we obtained that neutrino oscillations happen in time. The weakness of that method was that to get the stable oscillations picture, i.e. reproduce the Pontecorvo formula, one should prepare very broad initial wave packet. Physically it would correspond to a permanent, or rather long-continued, neutrino source.

One can also treat neutrino flavor oscillations in vacuum describing the propagation of neutrino mass eigenstates as the internal lines in a Feynman diagram [5, $\underline{6}, \mathbf{7}]$. Thus a Feynman propagator serves as a Green function in that approach. In that method one replaces flavor eigenstates with mass eigenstates at the neutrino emission and detection points. It is possible to say that one describes neutrino oscillations without resort to flavor neutrinos. It was found that transition and survival probabilities depend on the distance between emission and detection points. Although that approach seems to be quite natural it encounters some problems. For example, it is unclear (i) if different mass eigenstates should have equal energies, (ii) if one loses coherence when the energy is measured with high accuracy, (iii) if the usual Pontecorvo formula is unique because the non-standard oscillations formulae are possible in that approach (see the criticism in Ref. [14]).

In the present work we developed another approach to the neutrino flavor oscillations problem. In frames of our method we derived the coordinate dependent transition and survival probabilities. We examined the relaxation phenomena occurring when a neutrino signal arrives to a detector. It was revealed that the stable oscillations picture could be implemented if the time after the neutrino emission is much greater than the distance between a source and a detector. It means that the quantum mechanical transition probability formula is valid for a rather long-continued neutrino source. This result, in a sense, confirms the results of our previous works [15] (see also above). Note that one can come the similar conclusions basing on Ref. [27] where neutrino oscillations were studied on the basis of an exactly solvable model.

The weakness of the present method is that in various processes where a neutrino participates, particles belonging to different flavors can be emitted (see, e.g., Ref. [28]). For instance, there are the following decay channels of a charged pion: $\pi^{+} \rightarrow \mu^{+}+\nu_{\mu}$ and $\pi^{+} \rightarrow e^{+}+\nu_{e}$. Therefore in frames of our model the classical sources of both muon and electron neutrinos would correspond to this process. However the latter channel is suppressed by the factor $10^{4}$ [29]. Thus we can suppose that only one type of neutrino sources is presented. Nevertheless one can formally consider the sources of all neutrino species in frames our method. The amplitudes $f_{\lambda}^{(0) \mu}$ of the sources should be taken either from the quantum field theory of the neutrino emission or from an experiment.

Note that neutrino flavor oscillations in the model with a source and a detector were described in Ref. [30]. In that paper the source of particles was also assumed to be spatially localized. However its time dependence was chosen to be gaussian that is different from our approach.
Summarizing we can say that our method for the description of the flavor neutrinos evolution can be useful when we have spatially localized neutrino sources which start to emit neutrinos at some moment of time. One of the possible examples is the neutrinos emission in a supernova explosion.

\section{Acknowledgments}

The work has been supported by the Academy of Finland under the contract No. 108875 and by the Conicyt (Chile), Programa Bicentenario PSD-91-2006. The author is thankful to the Russian Science Support Foundation for a grant as well as to Jukka Maalampi (University of Jyväskylä) and Walter Grimus (University of Vienna) for helpful discussions.

\section{APPENDIX A: EVALUATION OF INTEGRALS}

It is interesting to evaluate the inexactitude which is made when we approach to the limit $x_{m} \rightarrow \infty$ in Eq. (2.13). Let us discuss two functions

$$
\begin{aligned}
F(r, t) & =\int_{0}^{x_{m}} \mathrm{~d} x \frac{J_{1}(m x)}{\sqrt{r^{2}+x^{2}}} e^{\mathrm{i} E \sqrt{r^{2}+x^{2}}} \\
& =\int_{0}^{y_{m}} \mathrm{~d} y \frac{J_{1}(\rho y)}{\sqrt{1+y^{2}}} e^{\mathrm{i} \mathcal{E} \sqrt{1+y^{2}}}, \\
F_{0}(r) & =\frac{1}{\rho}\left(e^{\mathrm{i} \mathcal{E}}-e^{\mathrm{i} P}\right) .
\end{aligned}
$$

These functions are proportional to $\mathcal{I}_{2}$ in Eqs. 2.13) and (2.14) respectively. In Eqs. (A1) and (A2) we use dimensionless parameters $\rho=m r, \mathcal{E}=E r=\gamma \rho$, $\gamma=E / m, y_{m}=\sqrt{(t / r)^{2}-1}$ and $P=\sqrt{\mathcal{E}^{2}-\rho^{2}}$.

In case we study neutrinos, we get that $\mathcal{E} \gg \rho \gg 1$ in almost all realistic situations. For example, suppose we study a neutrino emitted in a supernova explosion is our Galaxy. The typical distance is $r \sim 10 \mathrm{kpc}$. Taking $m \sim 1 \mathrm{eV}$ and $E \sim 10 \mathrm{MeV}$, we receive that $\mathcal{E} \sim 10^{35}$ and $\rho \sim 10^{28}$.

Basing on the analysis of Sec. II we can rewrite Eq. (A1) as

$$
\begin{aligned}
F(r, t) & =F_{0}(r)-\delta F \\
\delta F & =\int_{y_{m}}^{+\infty} \mathrm{d} y \frac{J_{1}(\rho y)}{\sqrt{1+y^{2}}} e^{\mathrm{i} \mathcal{E} \sqrt{1+y^{2}}} .
\end{aligned}
$$

Using the fact that $\rho \gg 1, y_{m} \gg 1$ and the representation for the Bessel function,

$$
J_{1}(z) \approx \sqrt{\frac{2}{\pi z}} \cos \left(z-\frac{3 \pi}{4}\right) \quad \text { at } \quad z \rightarrow+\infty
$$

we obtain for the function $\delta F$ in Eq. (A3) the following 


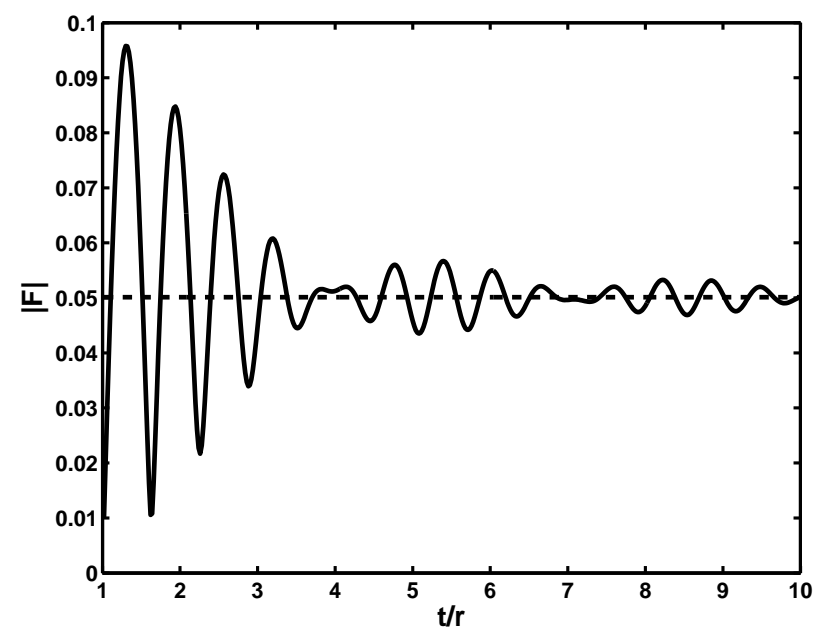

FIG. 1: The absolute values of the functions $F(r, t)$ and $F_{0}(r)$ versus $t$.

expression:

$$
\begin{aligned}
\delta F \approx & -\frac{1}{\sqrt{2 \pi \rho}}\left\{\operatorname{ci}\left([\gamma+1] \rho y_{m}\right)+\operatorname{ci}\left([\gamma-1] \rho y_{m}\right)\right. \\
& \left.+\mathrm{i}\left[\operatorname{si}\left([\gamma+1] \rho y_{m}\right)+\operatorname{si}\left([\gamma-1] \rho y_{m}\right)\right]\right\}
\end{aligned}
$$

where $\operatorname{ci}(z)$ and $\operatorname{si}(z)$ are cosine and sine integrals. Using the asymptotic expression,

$$
|\operatorname{ci}(z)| \sim|\operatorname{si}(z)| \sim \frac{1}{z} \quad \text { at } \quad z \rightarrow+\infty
$$

we obtain that the function $\delta F$ approaches to zero as $1 /\left(y_{m} \rho^{3 / 2}\right)$ at great values of $y_{m}$ and $\rho$. Note that this result remains valid for a particle with an arbitrary $\gamma$ factor, i.e. rapid oscillations of the function $\delta F$ will attenuate even for slow particles. This analysis substantiates the approximations made in Sec. II]

Finally let us illustrate the behavior of the functions $F(r, t)$ and $F_{0}(r)$. On Fig. 1 we present the absolute values of these functions versus $t$. This figure is plotted for $\rho=1$ and $\mathcal{E}=10$. The solid line is the absolute value of the function $F(r, t)$ and the dashed line $-F_{0}(r)$. As we mention in Sec. II the relaxation phenomena occur when $t \gtrsim r$. It can be also seen on Fig. 1, It is possible to notice that $|F(r, t)| \rightarrow\left|F_{0}(r)\right|$ at great values of $t$ as it is predicted in Sec. II]
[1] B. Aharmim, et al. (SNO Collaboration), Phys. Rev. C 72, 055502 (2005), nucl-ex/0502021 M. H. Ahn, et al. (K2K Collaboration), Phys. Rev. D 74, 072003 (2006), hep-ex/0606032; C. Arpesella, et al. (Borexino Collaboration), submitted to Phys. Rev. Lett., 0708.2251 [astro$\mathrm{ph}$.

[2] V. N. Gribov and B. Pontecorvo, Phys. Lett. B 28, 493 (1969); B. Kayser, Phys. Rev. D 24, 110 (1981).

[3] I. Yu. Kobzarev, et al., Sov. J. Nucl. Phys. 32, 823 (1980).

[4] E. K. Akhmedov, hep-ph/0610064.

[5] I. Yu. Kobzarev, et al., Sov. J. Nucl. Phys. 35, 708 (1982).

[6] C. Giunti, et al., Phys. Rev. D 48, 4310 (1993), hep-ph/9305276

[7] W. Grimus and P. Stockinger, Phys. Rev. D 54, 3414 (1996), hep-ph/9603430

[8] M. Blasone and G. Vitiello, Ann. Phys. 244, 283 (1995), Erratum ibid. 249 (1996) 363, hep-ph/9501263

[9] M. Blasone, et al., 0707.4476 [hep-ph].

[10] Y. F. Li and Q. Y. Liu, JHEP 0610, 048 (2006), hep-ph/0604069

[11] C. Y. Cardall, Phys. Rev. D 61, 073006 (2000), hep-ph/9909332

[12] A. D. Dolgov, et al., Eur. Phys. J. C 44, 431 (2005), hep-ph/0506203

[13] J. H. Field, Ann. Phys. 321, 627 (2006).

[14] M. Beuthe, Phys. Rept. 375, 105 (2003), hep-ph/0109119

[15] M. Dvornikov, Phys. Lett. B 610, 262 (2005), hep-ph/0411101 in Proceedings of the IPM school and conference on Lepton and Hadron Physics, Tehran, 2006, ed. by Y. Farzan, eConf C0605151 (2007), hep-ph/0609139 Eur. Phys. J. C 21, 2403 (2006), hep-ph/0601156 to be published in J. Phys. Conf. Ser., hep-ph/0708.2975; M. Dvornikov and J. Maalampi, Phys. Lett. B 657, 217 (2007), hep-ph/0701209 M. Dvornikov, J. Phys. G 35, 025003 (2008), 0708.2328 [hep-ph].

[16] N. N. Bogoliubov and D. D. Shirkov, Introduction to the Theory of Quantized Fields (Interscience Publishers, Inc., New York, 1960) 2nd ed., pp. 136-140.

[17] See pp. 648-653, in Ref. [16].

[18] C. Itzykson and J.-B. Zuber, Quantum Field Theory (McGraw-Hill, Inc., New York, 1980), pp. 682-698.

[19] J. Schechter and J. W. F. Valle, Phys. Rev. D 22, 2227 (1980).

[20] L. B. Okun', Leptons and Quarks (Nauka, Moscow, 1990), 2nd ed., pp. 36-42.

[21] Z.-Z. Xing, Phys. Lett. B 660, 515 (2008), 0709.2220 [hep-ph].

[22] M. Fukugita and T. Yanagida, Physics of Neutrinos and Applications to Astrophysics (Springer, Berlin, 2003), pp. 292-296.

[23] K. M. Case, Phys. Rev. 107, 307 (1957).

[24] M. I. Shirokov and V. A. Naumov, Concepts Phys. 4, 103 (2007), hep-ph/0611202.

[25] B. Pontecorvo, JETP 7, 172 (1958); for the contemporary description see pp. 286-287 in Ref. 22].

[26] J. Schechter and J. W. F. Valle, Phys. Rev. D 23, 1666 (1981); A. de Gouvêa, B. Kayser, and R. N. Mohapatra, Phys. Rev. D 67, 053004 (2003), hep-ph/0211394

[27] A. Ioannisian and A. Pilaftsis, Phys. Rev. D 59, 053003 (1999), hep-ph/9809503.

[28] R. E. Shrock, Phys. Lett. B 96, 159 (1980). 
[29] W. M. Yao, et al. (Particle Data Group), J. Phys. G 33,

hep-ph/9710289 1 (2006).

[30] K. Kiers and N. Weiss, Phys. Rev. D 57, 3091 (1998), 